\title{
Course training Market-Oriented research on information service platform based on RSS
}

\author{
Li Pan,Zhai Jun, Li Tao-Ying \\ Transportation Management College,Dalian Maritime University, Dalian, China \\ lipan_2013@163.com
}

Keywords: RSS; information service platform; information delivery; Course training Market

\begin{abstract}
It is essential for college students to participate in extra-curricular training for improving their competitiveness. However, there is large number of online educational institutions and numerous or types of courses is updated in real time. What else, colored leaflets about promotion are filled in every corner of the campus. So, how to collect effectively current and comprehensive information from web or leaflets is meaningful. This paper presents a Secondary Information Processing Platform, which uses RSS to extract, syndicate information, and re-display for users to browse. By collecting and reorganizing RSS course information, platform integrated courses and relevant comments into new RSS file for subscribing.
\end{abstract}

\section{Introduction}

Admittedly, in the 21st century we live in an era of information explosion. But we are forced to receive a "not" our information, such as leaflets, spam. Obviously, a lot of data meet the user' $s$ demand for the breadth of information, but at the same time, it affects the accuracy of information. Undoubted, this increases the cost of users' time to access the useful information. In the university, there are filled with colorful leaflets about course or training information almost everywhere.But, the article [1] use a large amount of data to prove the distribution of advertisement materials attracted hardly any participants and foresees personalized information delivery will be more effective and a higher cost-effectiveness ratio. And, it is time-consuming work to log in every education site. What is else, the comments from others with experience are helpful to make a decision.

RSS (Really Simple Syndication) is a means of organizing and simplifying current awareness efforts, which is becoming more and more popular among Internet users. The article [2] presents real examples of RSS feeds produced by leading publishers and shows that the benefits and effectiveness of those RSS feeds are directly related with the availability, quality and richness of its content. Especially, RSS is widely used for its efficient aggregation features, customization and timely access to information in which fields have higher requirements about information timeliness, [3] such as library fields and instant news fields [4] etc.

In this paper, the student was select as the target user, presents the establishment of a "real-time" and "customization" and "word of mouth" RSS-based information service platform. The platform will show gathered information in the way of new RSS format for users to subscribe. When there has any changes, users can access it timely, such as new courses, new comments or update courses and so on. What is else, this platform architecture can also be extended to other groups as well as other topics. This is significant for changing the traditional means of information dissemination.

\section{About RSS}

RSS (Really Simple Syndication really Simple Syndication), a machine-readable XML format for content syndication, [5] allows users to subscribe to the desired information and receive notification when new information is available. Users do not need to open each page to browse, search for news of interest, but the interest of the Rapid Active news presented to the user without human intervention. [6] 


\section{How RSS Works?}

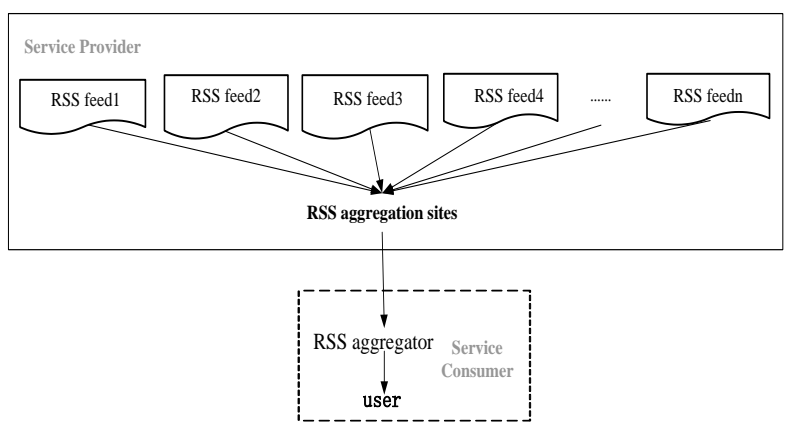

Figure 1.RSS Working Principle

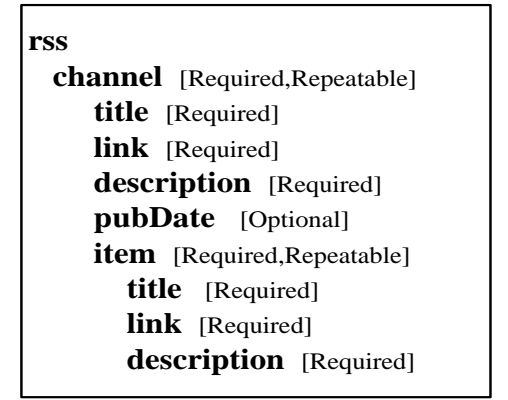

Figure 2.Structure of RSS 2.0 source

From Figure 1, we can conclude about how RSS works. First, create an RSS document and save it with an .xml or .rss extension. Then, upload the file to your website. Next, register with an RSS aggregator. Each day the aggregator searches the registered websites for RSS documents, verifies the link, and displays information about the feed so clients can link to documents that interest them.

RSS feed.A standard feed is a standard XML document. The document generally ends with rss, $\mathrm{xml}$ or rdf as a suffix. Figure 2 is structure of RSS 2.0 source.

A subscribe example.This is an RSS feed named example.xml extracting leaflet information. It was published to the local Apache server. So, it can be browsed by http://localhost/example.xml.

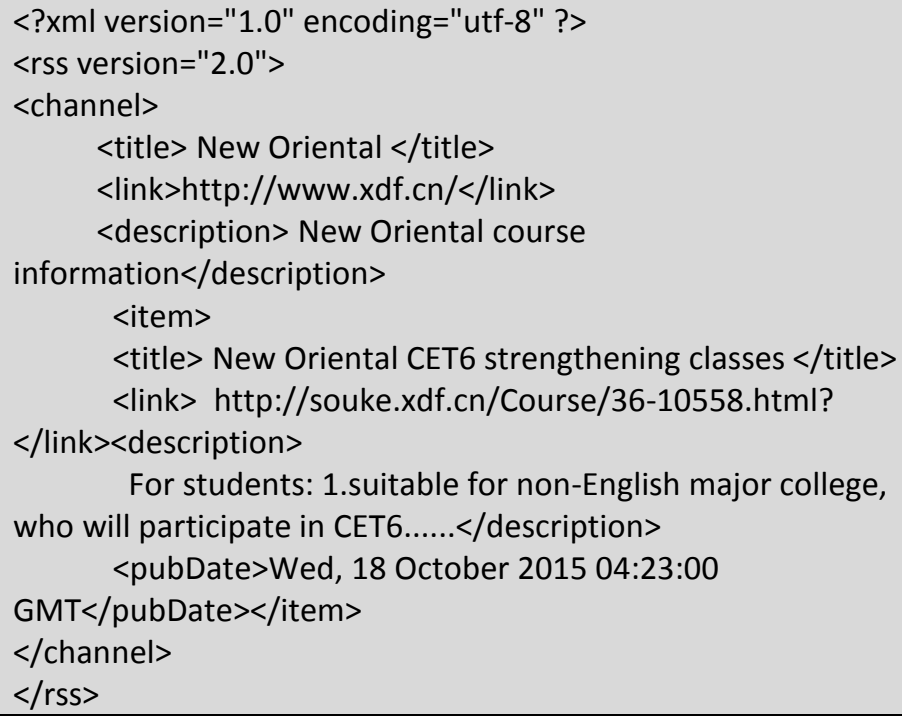

So far, an simple RSS feed was created successfully. The above feed should be subscribed to FeedDemon. When we entered the URL address, this subscribe process was finished. Else, user needs to set an update period to get news when it was updated.

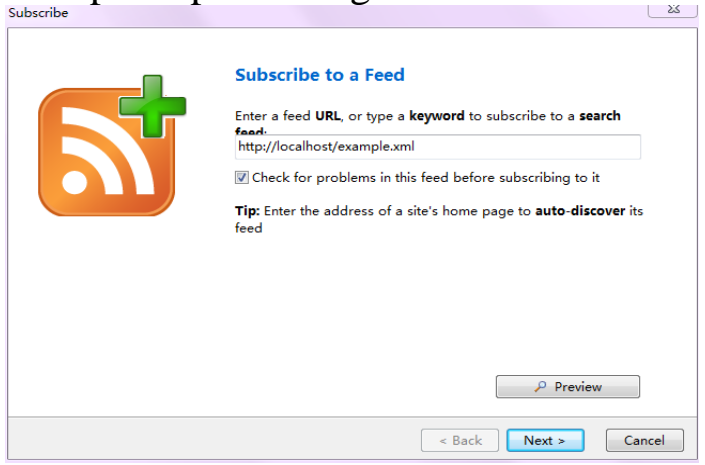

Figure 3.Page of subscription

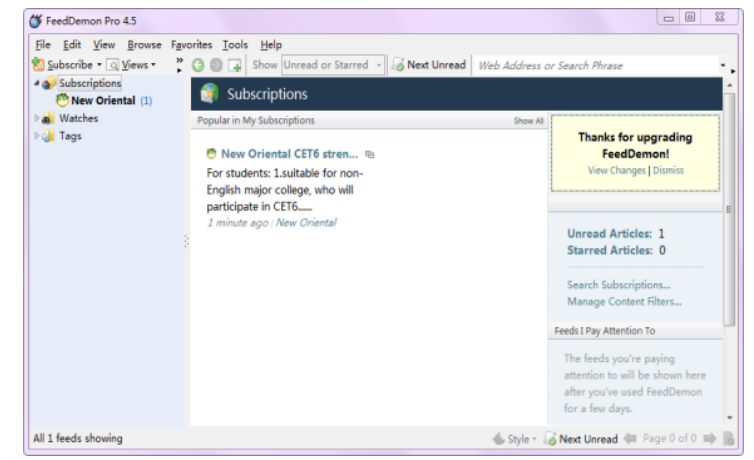

Figure 4.Page of viewing subscription 
Finally, users can easily access the information they are interested in. Once there have something modified we can get timely updates. It is worth emphasizing that any file with this mark RSS or XML button can be subscribed.

\section{Platform Introduction}

Training information is RSS file, also it refers to this platform can receive or process training information typed RSS format. Any non-RSS information only be preprocessed can flow this platform. We can learn from this article [7] about how to extract RSS feeds from HTML format. Here we, in accordance with its working mechanism, build a structure of platform as Figure 6.

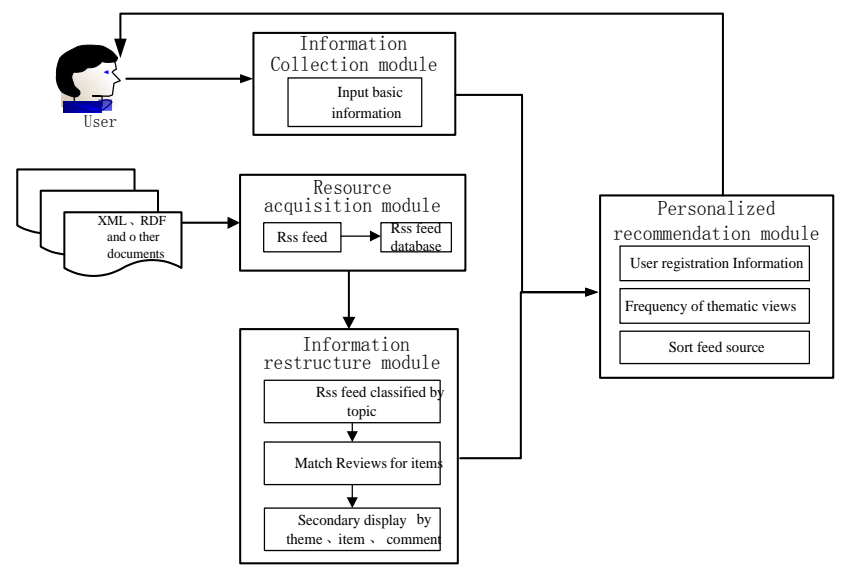

Figure 5.Structure of platform

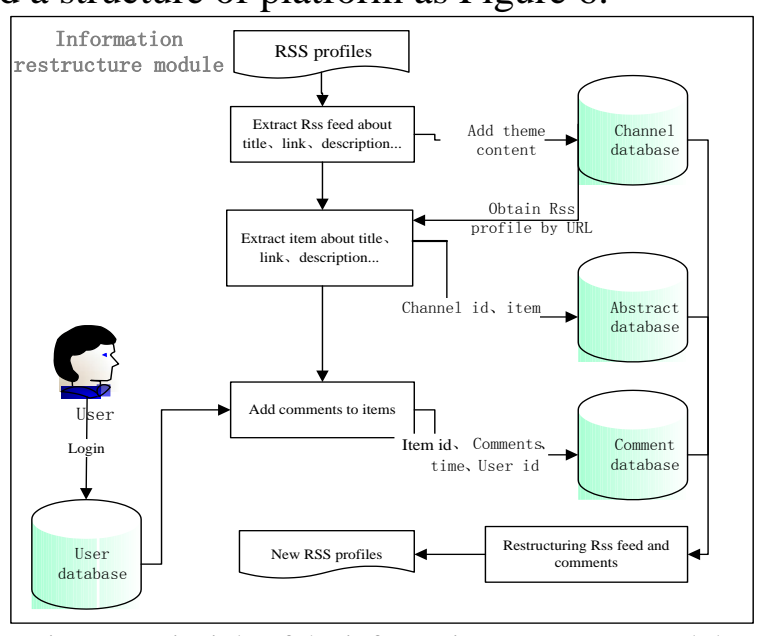

Figure 6.Principle of the information restructure module

The platform is divided into the following: Information collection module, Resource acquisition module, Information restructure module and personalized recommendation module. Information restructure module is most important for this platform,here, it will be introduced.

Information restructure module.The module's function is restructuring the original RSS file into new RSS files. This module is the core module of the platform, reflecting to connect users with various training information. [7] The figure7 is the operating principle of the information restructure module.

After the above series of processes, the new RSS structured schema is as follows. It is to find this new RSS structured schema is based on the above standard feed with <comment> element and sub-element added.

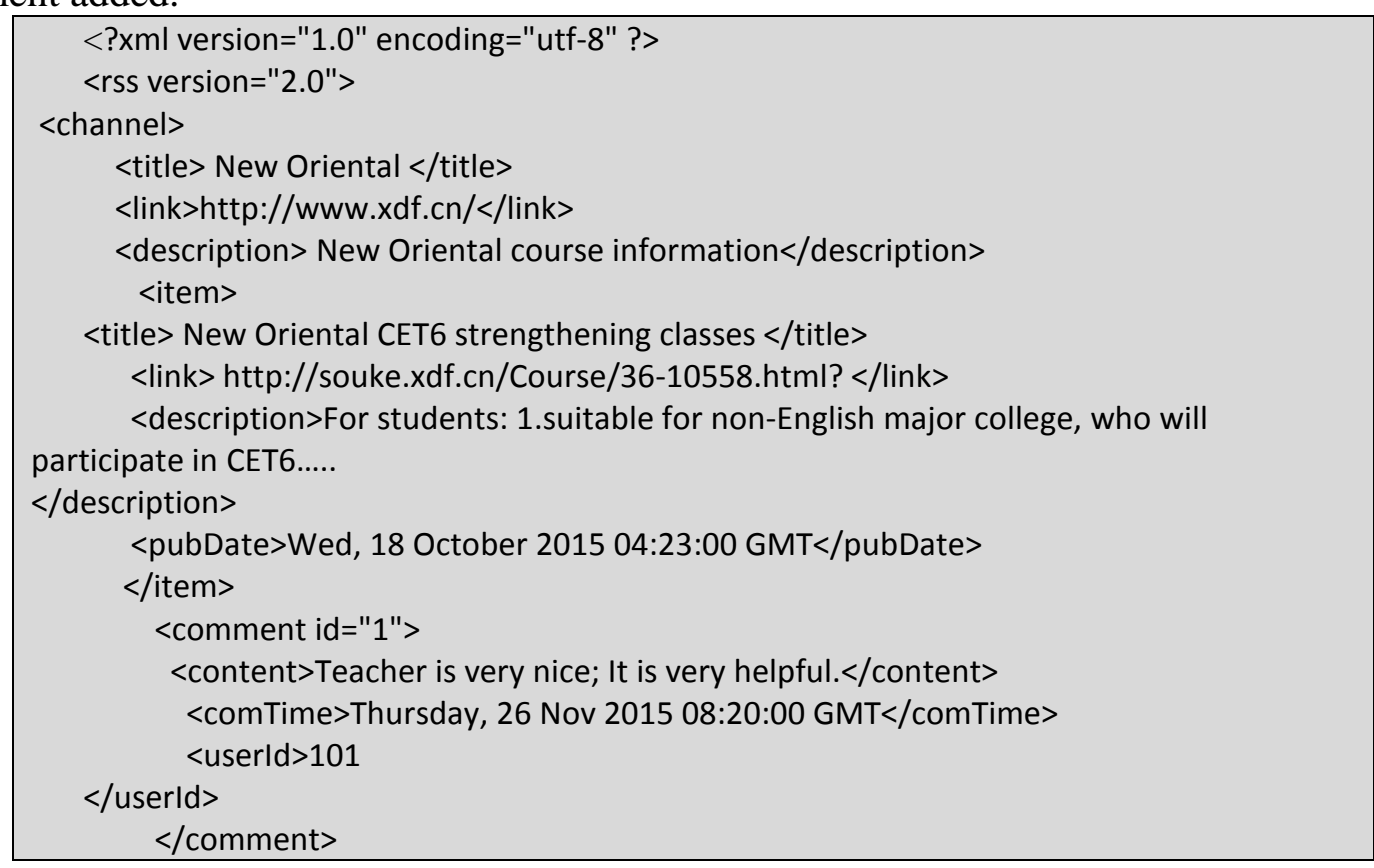




\section{Platform implemention and display}

The main page include a comment on the course we created is as follows.

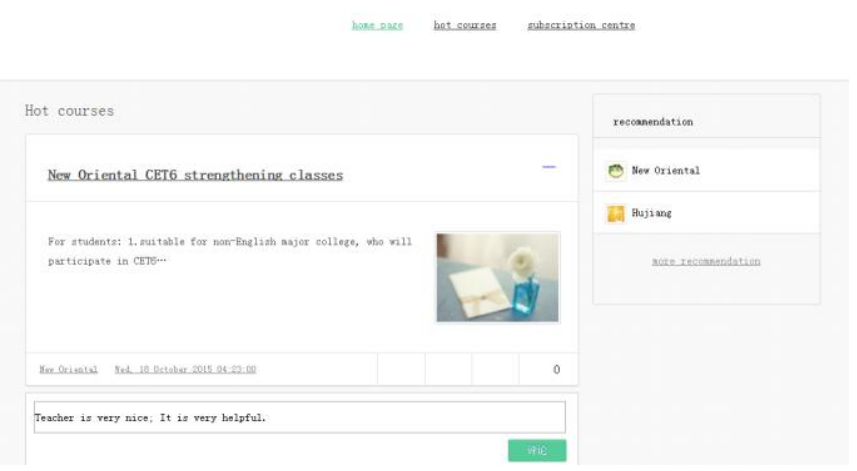

Figure 7.Main page

\section{Conclusions}

The platform is designed to optimize the traditional way to promote information with RSS technology that automatically delivers update course or comments information, posted by a publisher to subscribers' directly. In addition, this platform construction approaches can be widely promoted, in different fields. Scattered promotional information can be categorized using this efficient model to promote. Recommendation in this platform simply use a statistical analysis of knowledge, optimization of the functional modules would be the next focus of the work.

\section{Acknowledgment}

This work is partially supported by National Natural Science Foundation of China (No. 71271034), the Fundamental Research Funds for the Central Universities (No. 3132014080, No. 3132014307), National Natural Science Foundation of Liaoning (No. 2014025015), and Liaoning Academy of Social Sciences Fund (No. L13DGL060).

\section{References}

[1] Moessner M, Minarik C, Ozer F, et al. Effectiveness and Cost-effectiveness of School-based Dissemination Strategies of an Internet-based Program for the Prevention and Early Intervention in Eating Disorders: A Randomized Trial[J]. Prevention Science, 2015: 1-8.

[2] Santiago CHUMBE, Roddy MacLEOD. Marketing OA journals now that authors are customers: the role of RSS. LEARNED PUBLISHING VOL. 26 NO. 1(51-56) JANUARY 2013

[3] Barbara F. Schloman. Information Resources: Staying Current: What RSS can do for You [J]. Online Journal of Issues in Nursing, 2006, 11(1). 
[4] Ramli R M. A Study on the use of Facebook, RSS, Blogs and Twitter (Web2. 0) among selected academic libraries from 6 Gulf countries namely: Kingdom of Saudi Arabia, United Arab Emirates, Qatar, Bahrain, Oman and Kuwait[J]. QScience Proceedings, 2014.

[5] Rethlefsen M L, Rothman D L, Mojon D S. RSS[J]. Internet Cool Tools for Physicians, 2009: 77-86.

[6] Hammersley, B.: Content Syndication with RSS, Oreilly \& Associate, Inc. 1st edition(2003).

[7] T. Nanno and M. Okumura. HTML2RSS: Automatic Generation of RSS Feed Based on Structure Analysis of HTML Document. In Proc. Intl. Conf. on World Wide Web (WWW), pages 1075 - 1076, 2006. 